\title{
Educators' Perceptions of Uses, Constraints, and Successful Practices of Backchanneling
}

\author{
Cheri Toledo \\ Illinois State \\ Sharon Peters \\ Hebrew Academy
}

\begin{abstract}
This qualitative study sought to explore participants' perceptions of the impact of web-based backchanneling conversations in a variety of learning environments. Backchannels, forms of instant message conversations, take place during synchronous learning sessions. Online interviews with educators from Canada and the United States revealed their perceptions of the uses, constraints, and successful practices of backchanneling. Educators in the study saw backchanneling as a non-disruptive, non-subversive, collaborative activity that expanded participation and interactions; an approach applied with intentionality to enhance learning. Six themes emerged from the data: backchanneling for professional development and networking; backchanneling for engagement; constraints of backchanneling; changes in teacher and/or learner perspectives; examples of backchanneling in educational settings; and suggestions for successful backchanneling.
\end{abstract}

Keywords: web-based backchanneling; learning environments; professional development; networking 


\section{Educators’ Perceptions of Uses, Constraints, and Successful Practices of Backchanneling}

The convergence of mobile devices, ubiquitous wireless Internet access, and online communication tools has afforded the rising popularity of a phenomenon known as backchanneling. Originally perceived as an activity that was surreptitious, and possibly even subversive, it has become a common practice at conferences, meetings, and other presentationtype events. Web-based backchannels can be seen at educational technology events where they have shown to be valuable for educator professional development and growth. This study reports the findings of the in-depth interviews of backchannel-using educators. The emergent themes revolved around the uses, constraints, and successful practices of backchanneling.

\section{Defining Backchanneling}

The term backchanneling has been used extensively in linguistics when referring to the feedback loop of verbal (e.g., yes, uh huh) and nonverbal cues (e.g., head nods, smiles). A number of culture and language studies focus on this type of backchanneling (Kjellmer, 2009; Miyata \& Nisisawa, 2007; Heinz, 2003; Dixon \& Foster, 1998). Linguist Victor Yngve's (1970) originated the term back-channel in reference to conversational turn-taking. The advancement of information communication technologies in the last 40 years has digitized this practice of turntaking to include both face-to-face and virtual interactions.

However, for the purposes of this study, backchanneling is defined as instant messaging synchronously occurring between individuals and in groups using online tools during a live event. In this definition, the concept is often instantiated as a digital conversation taking place simultaneous to a live event. In addition, these types of sidebar discussions take place in a synchronous online environment using a variety of web-based tools, and many times include individuals who are not in the face-to-face setting.

\section{Problem, Purpose, and Research Questions}

Reports of backchannel use have appeared in blogs, list-servs, and the popular press. In addition, several conference proceedings reflect the developing body of knowledge regarding the use of backchanneling. There remains a gap in the literature concerning educators' perspectives of the use of the tool in learning settings. Therefore, the purpose of this interpretive qualitative study is to gain a deeper understanding of the instructional uses of backchanneling in learning sessions. Based on the theoretical construct and the literature, the following questions guided the research: (a) What are the educators' experiences using backchanneling in learning settings? (b) How are the educators using backchanneling in learning settings? (c) What are the patterns and themes related to the affordances and limitations of using backchanneling in learning settings?

\section{Theoretical Framework}

"Connectivism," coined as a learning theory for the digital age by Siemens (2004), provides a framework for understanding the phenomenon of backchanneling. Based on chaos, network, complexity, and self-organization theories, connectivism defines how people learn and construct knowledge. An important focal point of connectivism is the idea of representation points, or nodes, of potential information and knowledge. Connectivism describes the experience of learning in terms of new neural, conceptual, and external networks forming. This process occurs in complex, chaotic, and shifting spaces that are increasingly aided by technology. In fact, the backchannel network thrives in chaos. In addition to the collaborative processes (i.e., the compilation of collective knowledge) taking place in the backchannel, participants are also 
utilizing multi-nodal appliances to bring in new and supplemental information. It is these interactions with eclectic sources, modalities, and media that coincide with the premise of connectivism that knowledge resides in the networks we create. The theory of connectivism provides an excellent lens through which we can examine the phenomenon of backchanneling.

\section{Literature Review}

This review of literature focuses on the settings and uses of web-based backchanneling in learning sessions. Three distinct settings were mentioned in the literature: meetings, professional conferences, and educational settings. In addition, the literature regarding the practices of backchanneling will be addressed.

\section{Use in Meetings}

Shirky (2002) related his experience of using a chatroom during a 2-day brainstorming session and noted that participants were able to "add to the conversation without interrupting, and the group could pursue tangential material in the chatroom while listening in the real room" (para. 2). Backchanneling for individuals attending distributed meetings to engage in private messages to the other participants was reported by Brodt and Hoption (2005) and Yankelovich, McGinn, Wessler, Kaplan, Provino, and Fox (2005). Brodt et al. found that groups using the backchannel shared information that assisted them in making better decisions. Brodt et al. and Yankelovich et al. both reported that backchannels helped avoid interrupting the primary discussion and participants found the practice to have a positive impact on their meeting experiences.

\section{Use in Conferences}

Reinhardt, Ebner, Beham, and Costa (2009) collected data from face-to-face and remote attendees uses of backchanneling (using Twitter) at five different conferences. The researchers identified a variety of roles taken on by users, communication topics, and purposes for the backchannel. They found evidence that knowledge was enhanced through the use backchanneling. Weinburger (2004) and Crawford (2004), in reference to corporate settings, related the disruptive nature of the tool; even speaking about how conversations turned "adolescently defiant of authority" (Weinburger, para. 8) and were the "adult equivalent of notepassing during class" (Multitasking and Attention, para. 3). Jacobs and McFarlane (2005) observed that backchanneling disrupted the conventions of an academic conference and saw the need for the development of new skills and practices.

In 2005, McCarthy and Boyd analyzed Internet Relay Chat (IRC) logs from a conference and found that responses fell into four categories: greetings, logistics, work, and non-work and humor. In addition, the theme of "continuous partial attention" (p. 3) emerged to describe the lack of full attention that was paid to either discussion.

Harry, Gutierrez, Green, and Donath (2008) used a web-based backchannel system called backchan.nl to manage audience members' questions for presenters. The questions were voted on and the top-rated questions were projected during the presentation for all to see. This method made the backchannel overt rather than covert and elicited positive reactions from both presenters and users.

Siemens, Tittenberger, and Anderson (2008) stated that the increased utilization of technology (including backchanneling) has value for "extending activities and dialogue; selecting, scheduling, and promoting presentations; capturing content; supporting conversations; 
encouraging social networking; enabling tagging; fostering backchannel communication; and aggregating content" (p. 6). They asserted that through the use of backchanneling, conference participants are able to form their own social networks, enabling "In-depth online discussionsnot restricted by time and place-of key ideas, contrasting viewpoints, and multiple perspectives" (p. 7) that were archived and thus extending the learning.

\section{Uses in Educational Settings}

In 2001, Cogdill, Fanderclai, Kilborn, and Williams analyzed private transcripts of meetings and class sessions held in MUDs (Multi-User Dimensions). They identified five backchannel categories: process-oriented, content-oriented, participation-enabling, tangential and independent backchannel. Schick (2005) investigated student-to-student communication using text and audio with the goals of supporting "social interaction, collaborative learning, and engagement" (p. ii) in a lower division undergraduate computer science course. Students enjoyed the experience, were more engaged, and some of their feelings of isolation were alleviated; there was no significant increase in student learning.

Yardi (2006), found that the "Implementations of the backchannel will and should vary across different contexts and domains; teaching styles should take advantage of the social and educational affordances the backchannel offers; chatrooms should enable teacher selfassessment; a backchannel should encourage social interactions and community building; a backchannel etiquette will need to be developed” (p. 855-856).

\section{The Practice of Backchanneling}

Dr. Punya Mishra (2008a, 2008b), a professor at the Michigan State University, blogged his thoughts about using backchanneling in a doctoral course. After his initial use of the tool he was unsure of the connection between the primary class discussion and the backchannel. During the next class he led the students in a discussion of the backchannel discussion and then posted, "It seems to me that some kind of micro-blogging may be a great way of keeping the class engaged with the ideas etc. being discussed in class" (2008b, para. 5).

Along those lines, Scott Snyder (2009), a high school educator, blogged a series entitled Backchanneling Basics. In over a dozen posts, he discussed the practical uses, tools, and rationale for backchanneling in secondary education classrooms. This trend can also be seen in the February 2009 posting in which Olivia Mitchell shared How to Present While People are Twittering. Four months later, she wrote about How to Tweet During a Presentation (Mitchell, 2009, June). Taking the backchanneling process a step further than either of these was the SlideShare (http://www.slideshare.net) presentation by dmytro123 (2009) that included a rubric entitled "Self Assessment for Back Channel Contributions" (p. 3).

\section{Conclusion}

The growing literature on the uses of backchanneling is showing a progression from experimentation to integration. Available wireless technologies and their application to alternative styles of community building have ushered in a broader acceptance of backchanneling for a variety of teaching and learning environments. This study fills the gap in the research of backchanneling as it is used in educational settings. 


\section{Methodology}

This qualitative study is designed to explore the experiences of backchannel-using educators. This approach is based on the understanding that "meaning is socially constructed by individuals in interaction with their world” (Merriam, 2002, p. 3). In keeping with Merriam's characteristics of interpretive qualitative research, the following criteria were met: 1) the study focused on understanding the meaning that people have constructed about a phenomenon of interest; 2) the researchers are the primary instruments for data collection and analysis; 3) the research chiefly involves an inductive research strategy; and 4) the outcome of the study is descriptive in nature. To this end, the participants' own words are used to illustrate the findings.

\section{Data Collection}

In order to explore the participants' subjective experiences and interpretations of the use of backchanneling, in-depth interviews were conducted. Open-ended questions were grounded in the research questions, the theoretical framework, and the literature review. The following twelve questions provided a malleable structure for the interviews:

1. How would you define backchanneling?

2. What drew you to want to participate in this study?

3. What drew you to backchanneling?

4. Is it something that comes naturally to you?

5. Are you continuing to backchannel? What keeps you involved? (If not, why?)

6. Please describe some of your backchanneling experiences?

7. How would you describe backchanneling as an educational experience and what are the potential learning gains?

8. What are some of the constraints that might be prohibitive to this technique?

9. What circumstances would prevent you from backchanneling even if the opportunity available?

10. Have you used this technique with your students or seen it used with students?

11. Is this technique something we should be promoting as an educational

12. Is there anything you would like to add?

Table 1 shows how the interview questions align with each of the research questions.

\section{Table 1.}

Interview Questions Alignment with Research Questions

\section{Research Question}

Interview

Questions

$1,2,3,4,5,12$

1. What are the educators' experiences using backchanneling in learning 


\begin{tabular}{|l|l|}
\hline settings? \\
\hline 2. How are the educators using backchanneling in learning settings? \\
\hline 3. What are the patterns and themes related to the affordances and \\
limitations of using backchanneling in learning settings
\end{tabular}

There were several unique features about the approach to the data collection in this study. First, a call for potential participants was disseminated via Twitter - a social networking service. The researchers sent a message seeking experienced backchannel users to participate in the study. This resulted in a convenience sample of teachers, administrators, library media specialists, and education technology specialists. Second, the potential participants were contacted via email and directed to an online demographic survey (using SurveyMonkey). Third, the researchers created a GoogleDoc for each participant; GoogleDocs are a word processing tool that enables multiple editors. The interview questions were entered into the GoogleDocs, and each participant was given access and editing privileges to his/her document. The participants added information that was embellished during the interviews. Last, the audio interviews were conducted using Skype, an online video and instant messaging tool, and NiceCast software was used to record and archive them.

The digitally recorded interviews were transcribed into each participant's GoogleDoc. Due to the availability of the research questions and the transcripts of the interviews to the participants, member checks were the main method for insuring the rigor of the research; specifically credibility, dependability, and confirmability (Lincoln \& Guba, 1985). Each researcher independently conducted a content analysis of each interview transcript. The data coding process was aligned with Berkowitz' (1997) principles in which the following were identified and related to the research questions: common patterns and themes; pattern deviations and explanatory factors; and emergent stories. Once the themes and main ideas were identified, comparisons were made between the investigators' codings.

\section{Participants and Settings}

A group of educators, three from the Canada and fourteen from the United States, participated in this qualitative study on the uses of backchanneling in educational settings. The participants, 12 women and 5 men, were teachers, library media specialists, technology specialists, IT directors and support staff on the elementary, middle, secondary, college, and adult education levels.

After meeting face-to-face to develop the survey and interview questions, each researcher sent the initial call for participation out on Twitter. The participants were divided into two groups and each researcher conducted the individual Skype interviews with her group of educators. The interview sessions lasted 45-90 minutes. 


\section{Findings}

Close to 20 hours of interviews produced a rich set of data revealing how this group of educators perceived the uses, constraints, and successful practices of backchanneling. Six major themes and fourteen sub-themes (see Figure 1) emerged from the data.

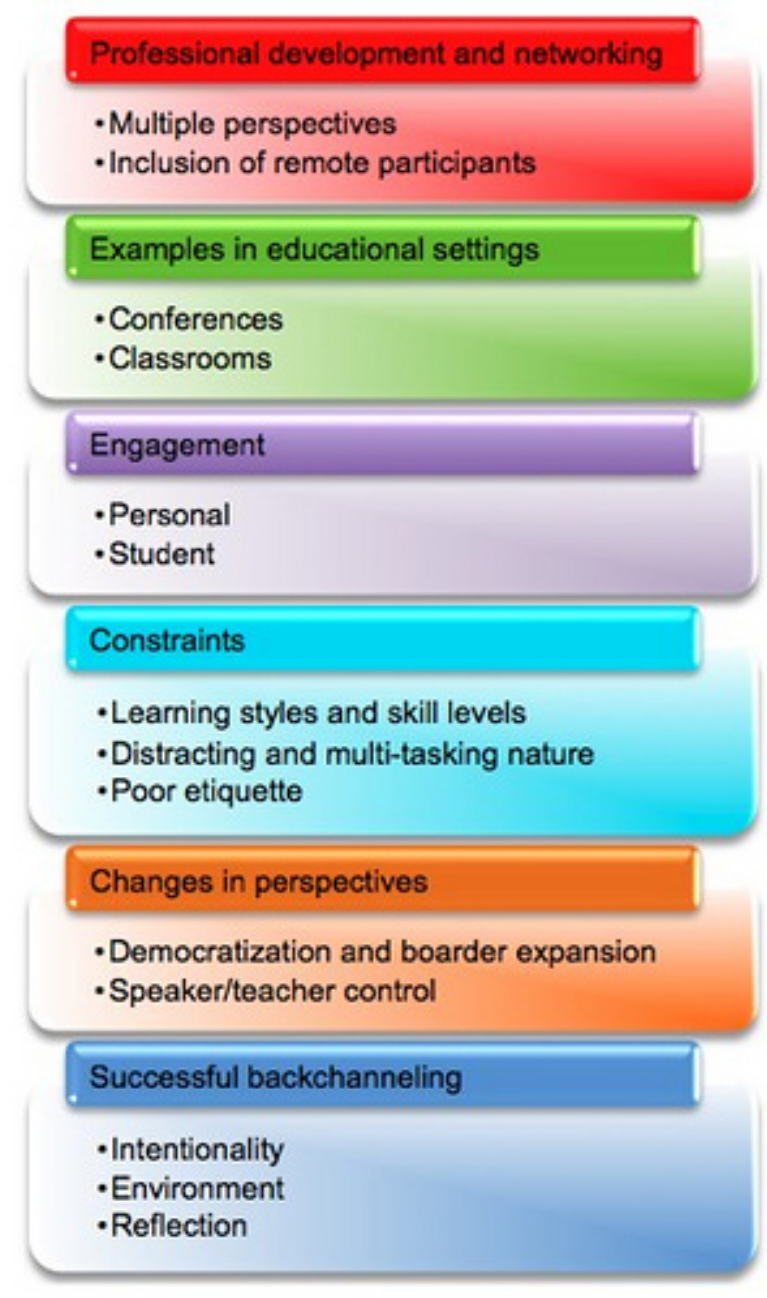

Figure 1. Themes and sub-themes

\section{Backchanneling for Professional Development and Networking}

It was the consensus of the participants that backchanneling provides unique opportunities for professional development. Each participant remarked upon this perceived benefit of backchanneling; in fact, one person referred to the practice as a "lifeline of learning" (Participant 2). When a backchannel was available during a presentation the conversations were extended in a variety of ways. The most obvious was the availability of the backchannel archive; it provided future access to the conversation, and sustained "the power of the question and ... the texture of the idea" (Participant 5). The conversation was also extended through the involvement of many voices. Most of the study participants talked of the inclusion of individuals who were not in the face-to-face meeting and the impact of this experience. This idea of multiple perspectives was a sub-theme that ran throughout the interviews. By garnering the perspectives of many people, the backchannel had the potential to become a Personal Learning Network 
(PLN) in itself and assisted the participants in expanding their global PLN. As participants talked about the use backchanneling for professional development and networking they emphasized the personal relationships and the creation of knowledge, not the technology itself, which thus reinforced the networking emphasis of connectivism.

\section{Examples of Backchanneling in Educational Settings}

The educators in this study brought with them a wide variety of backchanneling experiences. All of them had experienced backchannels as both face-to-face and virtual participants. The backchannel sessions that they reported varied in size from 1-200, lasting between 30 and 120 minutes, and used a variety of tools (Skype, UStream, Yugma, EdTechTalk chat, Elluminate, WebX, Chatzy, CoverItLive, GooglePresentation, FlashMeeting, Wimba, Meebo, and Twitter). The backchannels took place at conferences and in classroom settings.

Study participants reported being involved in backchannels in conjunction with the K-12 Online Conference, National Educational Computing Conference, Illinois Computing Educators Conference, Learning 2.0 Conference, the Discovery Conference and many more. The following relates some of the more unique experiences of the study participants.

Backchannels were used during in-class fishbowl discussions to engage the outer circle in the discussion. One participant created a collaborative exercise for American Literature students from two school districts, using the backchannel to discuss a YouTube video of Barack Obama's speech on race. Another educator set up a backchannel during a visit with an astronaut at the Space Station that enabled a school without a webcam or microphone to participate in the project. Backchanneling was used for quizzes, exit tickets, checks for understanding, and supplements to oral quizzes. It provided instant assessment and instant feedback, participation, and many backchannel tools enabled the chat to be archived. Some educators displayed the backchannel on a separate screen, which made the thinking and connection-making visible. It helped to keep the students on task and promoted the social construction of knowledge.

The participants also suggested hosting a guest speaker and linking classrooms from around the country or the world to interact with the guest. Using a backchannel with a WebQuest would expand the interactions, keep students on task, and "harness the power of the class" (Participant 7). Last, the use of backchannels in classrooms could serve as a tool for teaching digital safety and digital citizenship. The classroom backchannel provided a safe, monitored environment where students practice and learn appropriate online behaviors, especially in group chats. As Participant 7 stated, "Digital citizenship, like reading, needs to be [taught] across the curriculum." The appropriate and effective use of backchanneling can provide students with those opportunities.

\section{Backchanneling for Engagement}

Themes involving the increase of personal and student engagement through backchanneling emerged throughout the study. Several participants stressed that as a result of having used backchanneling frequently while attending presentations, they were disappointed when the opportunity to engage in a parallel discussion was not available. An increased level of connection to the speaker was noted. One participant stated, "Backchanneling unleashes the experience of the audience" (Participant 7). Participants recognized the value of engaging with the presenter's ideas and with other backchannelers during the presentation rather than waiting until afterward - or possibly never. Some complained that since they had begun the practice, 
they found it more difficult to remain passive audience members - instead, they started their own backchannels to increase their own engagement.

In looking at student engagement, participants emphasized that backchanneling provide students with "a greater sense of ownership over [their] learning" (Participant 4), resulting in increased engagement as the students chose their levels of involvement; "they come alive in a backchannel" (Participant 9). The backchannel provided students with a place to find their voices; and emphasized active listening and informal learning. Students experienced the instantaneous responses with which they had become accustomed; they were interacting "with the content as it's happening” (Participant 10). In addition to the increased engagement that occurred as students use the backchannel, they were also making connections - connections to the text and to their lives.

Another iteration of engagement involved the emergence of the marginalized students in the backchannel conversations. Shy and introverted students were reported to be very involved in the backchannel. It provided a more comfortable mode of communication for the introverted and shy students by creating a "very non-threatening way to throw out ideas and test the waters" (Participant 7).

\section{Constraints}

Several participants spoke of backchanneling not working for all learning styles. It was suggested that linear thinkers would struggle, but that kinesthetic, hands-on learners would find the practice more suited to their learning preferences. It is important to note, "not everyone likes [backchanneling] or is good at it" (Participant 11). Just as with face-to-face group discussions there will be those who will be comfortable, those who will be passive, and those who will dominate - some of these factors are related more to personality than learning style. The backchanneling process is further complicated if participants' computer skill levels are not up to the challenge. This variance of preferences and skills will impact the potential and the effectiveness of the backchannel.

While the study participants admitted that backchanneling could be a distracting activity, several educators talked about how the multi-tasking nature of backchanneling suits their personal learning and interaction styles. The ability to participate in a conversation, access other resources, and bring information back into the conversation was mentioned by many as a strength. However, there was a concern about the difficulties of focusing on so many conversations at once. Some may be able to focus on the primary conversation, some on the backchannel, but not on both. This idea of "not being fully in the moment" and giving "continuous partial attention" (Participant 11) to either conversation highly impacted one's ability to process both the presentation and the backchannel information. Off-topic posts in the backchannel, evident in the literature and the study, intensified the distractive characteristics of the tool.

Poor etiquette was identified as another constraint. It was reported the backchannel could be "disruptive at times; [there is a] need for etiquette. If it's [etiquette] not there it can be disruptive and confusing" (Participant 13). As indicated in the literature, the educators in this study acknowledged the negative impact of participants' inappropriate behaviors on the effectiveness of the backchannel. Participants identified this area as a potential major constraint that influenced their choice of using backchanneling within their educational settings. One participant stated that she would not choose to use backchanneling if she had a group of students 
who were disruptive and could not follow instructions. Two of the participants noted that the issue of control had to be taken into consideration. It was acknowledged that a good deal of control of behavior is relinquished when students, or even adult participants, are participating in a backchannel. One participant noted that most educators are very reluctant to give up control of their students and, for this reason, she found it doubtful that the practice of backchanneling would be readily adopted in the short term (Participant 4).

Study participants indicated that the constraints of backchanneling could slow its acceptance in the education culture. Several mentioned the importance of a change in the social norms; the need for a change in the teacher-speaker/learner-participant paradigm. The predominance of the hierarchical approach to teaching and learning does not lend itself to successful backchanneling. When speakers/teachers move from a position of total control over the information stream, the social structure is changed and the dynamic is democratized giving backchannelers new levels of freedom. Backchanneling "that puts [participants] on a more equal footing with the presenter ... and ... gives [them] more power than [ever] before" (Participant 4).

This entire process expands the borders of interaction and learning by enabling participants to share their experiences, knowledge, questions, and thoughts. Democratization of the learning environment occurs with face-to-face participants and those at a distance. Bringing participants in from remote locations extends the conversation and provides a variety of perspectives. As a result of all the interaction occurring during these open sessions, knowledge is socially constructed. "There's a trust and a belief that over time people's ideas will connect and solidify” (Participant 5). This is a huge shift for many involved in education (teachers, administrators, students, and parents). As educators see the empowerment that can occur as students are given more control they will be able to come to the conclusion that Participant 4 shared, "it's not your lesson, it's their learning."

\section{Suggestions for successful backchanneling}

The study participants emphasized three processes that contribute to successful backchanneling sessions: intentionality, environment, and reflection. Intentionality begins with taking the time to plan and planning begins with identifying the desired outcomes and purposes for the backchannel. It is imperative to set clear expectations and formalize the backchanneling methodology. Teachers and speakers must determine if a backchannel will achieve the goals that they have for their students or audience.

Once the goals are set, the organizer must plan for different levels of engagement and variety of interactions. It is important to note that the level of planning is directly proportional to the number of participants and sites involved. It is important to determine the most appropriate backchannel configuration for the target audience; e.g., audio, video, specific. For some situations, such as the fishbowl exercise, keeping the backchannel groups small ensures that everyone makes a strong contribution. For larger speaker-centered sessions, the environmental issues will differ. Anticipating the size of a group will help determine how much moderation is required.

Another important factor for a successful backchanneling session is the skill levels of the participants. It was the consensus of the participants that backchanneling involves a new technology skill set. When we are involved in informal backchannels, such as those that occur spontaneously during a presentation, coaching or peer-mentoring often takes place as participants help newcomers become familiar with the features of the online platform or aid in explaining 
background context to the presentation. In educational settings, however, teachers must include time for skill-building activities in their plan. Research participants indicated that the skills for a successful backchannel session involve keeping on topic, being able to ask appropriate questions, bringing in resources (links and information), and synthesizing the conversations (both the main discussion and the backchannel). It was recommended to provide students at first with a private space where they can experiment, make mistakes, and learn to use the backchannel correctly.

According to the study participants, several environmental factors will ensure a successful backchanneling experience for learners. First, it was suggested that each session include at least one moderator (some referred to this person as a rudder). The moderator makes sure that participants are exhibiting good etiquette, that the conversation stays on topic, and that the speaker receives information and feedback from the backchannel. The moderator should have the ability to summarize and synthesize pertinent information and share that with the leader of the primary discussion. Others talked of including a "Web jockey"; someone who listens to the primary conversation, watches the backchannel conversation, and then provides links to online information in the backchannel. This person is connecting the conversations to one another through the vast resources on the web. "Facilitating a backchannel is a skill in itself" (Participant 7). Therefore, it is imperative for moderator and web jockey skills to be taught the skills of these roles.

It is important to emphasize one topic that was referred to by almost every participant, netiquette. Netiquette is online "conduct that is appropriate and courteous" to other users (Wiktionary, n.d.). As stated earlier, backchanneling provides opportunities to incorporate digital ethics and appropriate uses of online communication into students' learning habits. Educators can create a backchannel to talk about and practice backchannel etiquette. It imperative to talk with students about the ethical issues of their posts and real-world perceptions. At the same time, teachers must provide them with clear expectations of appropriate posts and acceptable behavior.

Creating an environment also involves choosing the appropriate backchannel configurations and tools. For some situations, such as the fishbowl exercise, keeping the backchannel groups small ensures that everyone makes a strong contribution. For larger speakercentered sessions, making sure that there are enough moderators and jockeys for the group size. Different tools provide different outcomes. Some tools can be embedded in another technology (e.g., Meebo in a wiki); others can be archived, making available synchronized recordings the full audio and text (e.g., Elluminate, UStream).

Lastly, it is important for teachers and students to reflect on the backchannel experience. The potential for rich learning is enhanced when students (and audiences) have opportunities to review the backchannel transcript to reinforce their learning. One of the beauties of having an archive, especially in conjunction with the audio, is the ability to re-experience the session - to say nothing of the availability of the session to those who could not participate in real time. Teachers should use the time of reflection to determine the effectiveness of the backchannel in meeting the original objectives, determine needed changes, and make adjustments for future sessions.

\section{Conclusions}

According to the educators in this study, backchanneling is an effective learning tool that has many applications within academic environments. All of the participants saw backchanneling as an unparalleled tool for powerful interactions and resources transforming their learning and 
professional development. They also reported numerous examples of methods enabling students to benefit from well-planned backchanneling sessions that provide opportunities for students to participate in "traditional learning tasks [such as] reading, comprehending, understanding, and defending" (Participant 3). Also within this study, the notions of intentionality, netiquette, and reflection emerged as common themes among the participants. Careful planning was shown to help facilitate successful sessions. A few of the participants spoke of the need to grow the skills of digital netiquette and backchanneling experiences afford those opportunities. And finally, powerful reflection takes place when participants have opportunities to articulate their ideas during a backchanneling session and later review the archives. No longer viewed as a disruptive, subversive tool, effective backchanneling can provide a collaborative activity that expands engagement and learning - empowering participants and students to take control of their own learning. 


\section{References}

Berkowitz, S. (1997). Analyzing qualitative data. In J. Frechtling, L. Sharp, and Westat (Eds.), User-friendly handbook for mixed method evaluations (Chapter 4). Retrieved from National Science Foundation website http://www.nsf.gov/pubs/1997/nsf97153/chap_4.htm

Brodt, S., \& Hoption, C.(2005). Whispering in cyberspace: The effects of private communication on decision processes in distributed groups. Social Science Research Network Working Paper Series.

Cogdill, S., Fanderclai, T. L., Kilborn, J., \& Williams, M. G. (2001). Backchannel: Whispering in digital conversation. In Proceedings of the 34th Annual Hawaii International Conference on System Sciences.

Crawford, W. (2004, July/August). May I have your attention please? EContent. Retrieved from http://www.econtentmag.com/Articles/Column/DisContent/MayI-Have-Your-A...

Dixon, J. A., \& Foster, D.H. (1998). Gender, social context, and backchannel responses. The Journal of Social Psychology, 138(1), 134-136. Retrieved from ProQuest Education Journals. (Document ID: 27049343).

dmytro123. (2009). Backchanneling. Retrieved from http://www.slideshare.net/dmytro123/ backchanneling-1554084

Harry, D., Gutierrez, D., Green, J., and Donath, J. (2008). Backchan.nl: Integrating backchannels with physical space. In CHI '08: CHI '08 extended abstracts on Human factors in computing systems, 2751-2756. New York: ACM Press.

Heinz, B. (2003). Backchannel responses as strategic responses in bilingual speakers' conversations. Journal of Pragmatics, 35(7), 1113-1142.

Jacobs, N. and McFarlane, A. (2005). Conferences as learning communities: some early lessons in using 'back-channel' technologies at an academic conference - distributed intelligence or divided attention? Journal of Computer Assisted Learning, 21(5), 317-329.

Kjellmer, G. (2009). Where do we backchannel?: On the use of mm, mhm, uh huh and such like. International Journal of Corpus Linguistics, 14(1), 81-112.

Lincoln, Y. S., \& Guba, E. G. (1985). Naturalistic inquiry. Beverly Hills, CA: Sage Publications, Inc.

McCarthy, J. \& Boyd, D. (2005). Digital backchannels in shared physical spaces: Experiences at an academic conference. Proceedings of the SIGCHI Conference on Human Factors in Computing Systems (CHI ’05) Extended Abstracts. New York: ACM Press.

Merriam, S. B. (2002). Qualitative research in practice: Examples for discussion and analysis. San Francisco CA: Jossey-Bass.

Mishra, P. (2008a). Microblogging in the classroom. Punya Mishra's Web. Retrieved from http://punya.educ.msu.edu/2008/11/12/microblogging-in-the-classroom/

Mishra, P. (2008b). Microblogging in the classroom II. Retrieved from http://punya.educ.msu.edu/2008/11/20/microblogging-in-the-classroom-ii/

Mitchell, O. (2009, February). How to present while people are twittering. Pistachio. Micro sharing. Macro results. Retrieved from http://pistachioconsulting.com/twitter-presentations/ 
Mitchell, O. (2009, June). How to tweet during a presentation. Speaking about presenting: Help with your next presentation from Olivia Mitchell. Retrieved from http://www.speakingaboutpresenting.com/twitter/tweet-during-presentation/

Miyata, S., \& Nisisawa, H. Y. (2007, July). The acquisition of Japanese backchanneling behavior: Observing the emergence of aizuchi in a Japanese boy. Journal of Pragmatics, 39(7), 1255-1274. Retrieved from Science Direct database.

Reinhardt, R., Ebner, M., Beham, G., \& Costa, C. (2009). How people are using Twitter during conferences. Published in Creativity and innovation competencies on the web. HornungPrähauser, V., \& Luckmann, M. (Ed.). Proceeding of 5. EduMedia conference, p. 145-156, Salzburg.

Schick, R. J. (2005). A study of student conversation in text and audio during webcast lectures. Unpublished master's thesis. University of Toronto: Toronto, Ontario.

Shirky, C. (2002, Dec. 26). In-room chat as a social tool. O'Reilly OpenP2P.com. Retrieved from http://openp2p.com/lpt/a/3071

Siemens, G. (2004). Connectivism: A learning theory for the digital age. Retrieved from http://www.elearnspace.org/Articles/connectivism.htm

Siemens, G., Tittenberger, P., \& Anderson, T. (2008, March/April). Conference connections: Rewiring the circuit. Educause Review, 43(2). Retrieved from http://www.educause.edu/EDUCAUSE+Review/EDUCAUSEReviewMagazineVolume43/C ... 162675

Snyder, S. (N.d.). Backchanneling basics. Integrating Tech. Retrieved from http://thespian70.blogspot.com/search/label/backchannel

Weinburger, D. (2004, May). The backchannel world. KM World. Retrieved from http://www.accessmylibrary.com/coms2/summary_0286-21257590_ITM

Wictionary. (n.d.). Retrieved from http://en.wiktionary.org/wiki/netiquette

Yankelovich, N., McGinn, J., Wessler, M., Kaplan, J., Provino, J., and Fox, H. (2005). Private communications in public meetings. In CHI '05: CHI '05 extended abstracts on Human factors in computing systems, 1873-1876. New York: ACM Press.

Yardi, S. (2006). The role of the backchannel in collaborative learning environments. In ICLS '06: Proceedings of the 7th international conference on Learning sciences, pages 852-858. International Society of the Learning Sciences.

Yngve, V. (1970). On getting a word in edgewise. Papers from the Sixth Regional Meeting of the Chicago Linguistic Society, 567-577. 